\title{
Nefroangiosclerosi maligna: un caso a sorpresa
}

\author{
A. Capitanini, I. Petrone
}

\author{
U.O. Nefrologia ASL3, Ospedale Civile di Pescia (Pistoia)
}

Maschio di 39 anni ricoverato perché, a seguito degli accertamenti richiesti per il rinnovo della tessera di caccia, gli viene riscontrata insufficienza renale (creatininemia $3.7 \mathrm{mg} / \mathrm{dL}$ ) e ipertensione arteriosa di grado severo (PA 210/130 mmHg).

Dall'anamnesi: familiarità per ipertensione arteriosa, fuma circa 20 sigarette al giorno, gioca a calcio a livello amatoriale. Riferito episodio di tipo sindrome nefritica postinfettiva (varicella) in età infantile. Non altri significativi problemi di salute; non assume terapie.

All'ingresso il paziente è asintomatico. Esame obiettivo: peso $88 \mathrm{~kg}$, altezza $190 \mathrm{~cm}$, PA 210/130 $\mathrm{mmHg}$; azione cardiaca ritmica normofrequente, non soffi cardiaci, murmure vescicolare presente su tutto l'ambito polmonare, non edemi declivi, polsi arteriosi presenti, non soffi vascolari addominali, cute e mucose normocromiche e normoidratate.

Gli esami biochimici: urea pl. $107 \mathrm{mg} / \mathrm{dL}$, creatininemia $3.4 \mathrm{mg} / \mathrm{dL}$, e-GFR secondo formula CKP-EPI pari a 18 $\mathrm{mL} / \mathrm{min}$, sodiemia $145 \mathrm{mEq} / \mathrm{L}$, potassiemia $3.4 \mathrm{mEq} / \mathrm{L}$, calcemia totale $8.6 \mathrm{mg} / \mathrm{dL}$, uricemia $7.6 \mathrm{mg} / \mathrm{dL}$, colesterolo totale $179 \mathrm{mg} / \mathrm{dL}$, trigliceridi $130 \mathrm{mg} / \mathrm{dL}$, bilirubina $0.9 \mathrm{mg} / \mathrm{dL}, \mathrm{GOT} 16 \mathrm{U} / 1$, GPT $12 \mathrm{U} / 1$, attività protrombinica 97\%, INR 0.96, PTT 29 sec, tempo di emorragia 1 min e $40 \mathrm{sec}$, VES $18 \mathrm{~mm}$, PCR $2.08 \mathrm{mg} / \mathrm{L}$.

IgG $1159 \mathrm{mg} / \mathrm{dL}$, IgA $247 \mathrm{mg} / \mathrm{dL}, \operatorname{IgM} 90 \mathrm{mg} / \mathrm{dL}$, C3 $108 \mathrm{mg} / \mathrm{dL}, \mathrm{C} 436 \mathrm{mg} / \mathrm{dL}$, ANA neg, p-ANCA neg, cANCA neg.

fT3 $2.8 \mathrm{pg} / \mathrm{mL}$, fT4 $1.3 \mathrm{ng} / \mathrm{mL}$, TSH $5.9 \mathrm{mUI} / \mathrm{mL}$, aldosterone $283 \mathrm{pg} / \mathrm{mL}$, renina $36 \mathrm{pg} / \mathrm{mL}$.

Esame urine: $\mathrm{pH}$ 5.5, peso specifico 1006, proteine 150 $\mathrm{mg} / \mathrm{dL}$, glucosio assente, emoglobina $0.06 \mathrm{mg} / \mathrm{dL}$; sedimento con alcuni cilindri ialini, 5 globuli rossi pcm.

Diuresi $1.500-2.500 \mathrm{~mL}$, sodiuria $21 \mathrm{mEq} / 24 \mathrm{~h}$, potassiuria $47 \mathrm{mEq} / 24 \mathrm{~h}$, proteinuria $3.3 \mathrm{~g} / 24 \mathrm{~h}$.

Ecografia renale: "reni in sede con diametro longitudinale di circa $12 \mathrm{~cm}$ bilateralmente, corticale finemente ipe- recogena, spessore corticomidollare medio di circa $20 \mathrm{~mm}$, indici di resistenza lievemente aumentati (IR 0.66-0.72). Non calcoli, cisti o idronefrosi. Aorta ectasica in tutto il decorso addominale con diametro traverso 2.0-2.5 cm. Non stenosi emodinamiche sugli assi arteriosi renali, né anomalie vascolari intraparechimali ecorilevabili" (Fig. 1).

Ecocardiogramma: "ipertrofia concentrica del ventricolo sx (SIV $18 \mathrm{~mm}$ ) e dimensioni cavitarie aumentate (61 $\mathrm{mm}$ ), normale performance sistolica (EF 60\%)”.

Fundus oculi: "edema bilaterale della papilla".

Holter pressorio: "valori sistodiastolici elevati in assenza di ritmo circadiano, profilo non dippers" (Fig. 2).

È stata istituita un'adeguata terapia antipertensiva a base di calcio antagonisti, alfa litici, ace inibitori e beta bloccanti e, nel sospetto di una patologia glomerulare rapidamente evolutiva, il paziente è stato sottoposto, dopo 4 giorni di ricovero, ad agobiopsia renale. Al momento della biopsia la pressione arteriosa era 160/100 $\mathrm{mmHg}$. La manovra bioptica si è complicata con ematoma perirenale di notevoli dimensioni (9x4x7 cm) (Fig. 3), che si è sviluppato in circa 48 ore e ha indotto anemizzazione asintomatica, fino a valori di emoglobina di $8 \mathrm{~g} / \mathrm{dL}$, che si è progressivamente risolta senza necessità di emotrasfusioni.

Reperto istologico: MICROSCOPIA OTTICA frustolo agobioptico renale corticale con 23 glomeruli dei quali 8 interamente scleroialini, dei restanti di volume normale 3-4 mostrano ispessimento fibroso segmentale della capsula di Bowman, aree di ialinosi e proliferazione mesangiale in 2-3 glomeruli. Grave danno tubulo interstiziale con diversi infiltrati infiammatori linfocitari ed aree irregolarmente estese di fibrosi, atrofie tubulari e diverse dilatazioni pseudocistiche. Gravissimo danno vascolare caratterizzato da ispessimento della parete, sclerosi ed iperplasia intimale di vario grado con riduzione fino alla scomparsa del lume, presenza di diversi aspetti di tipo "onion like" di rami delle arterie interlobulari, gravissi- 


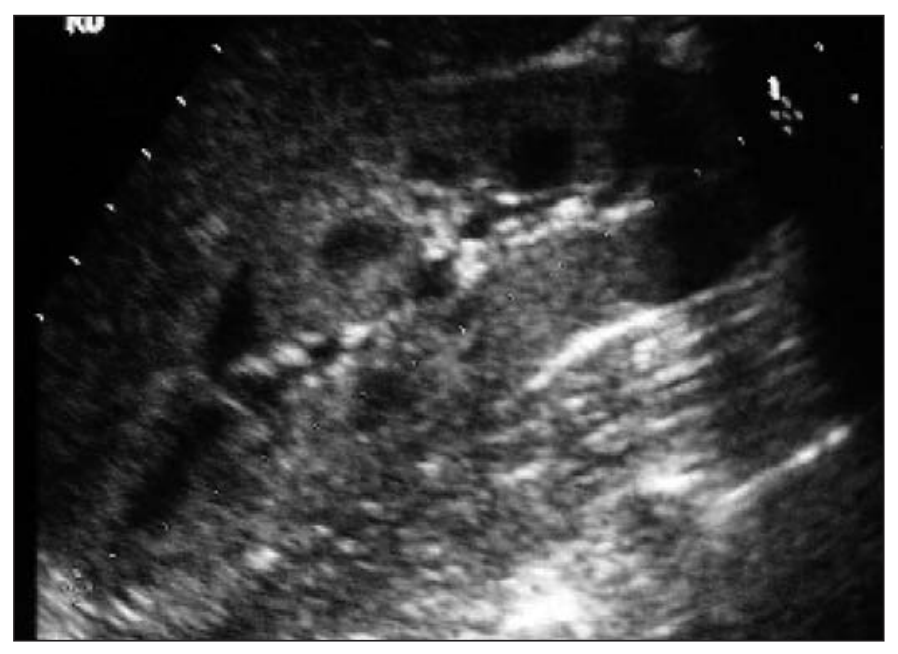

Fig. 1 - Rene di circa $11 \mathrm{~cm}$ con iperecogenicità corticale.

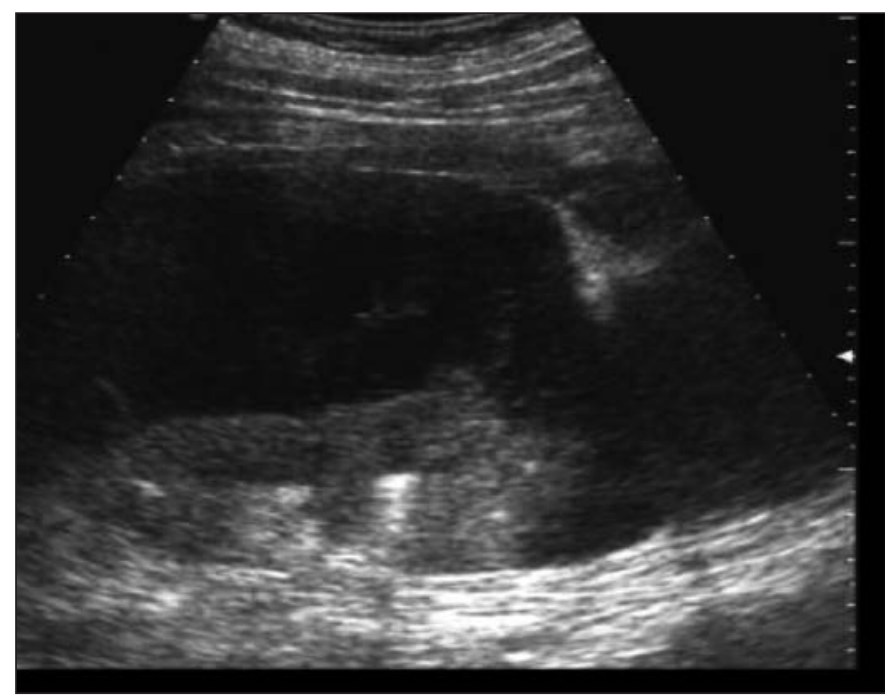

Fig. 3 - Grossa emorragia post bioptica perirenale sx.

ma arteriosclerosi delle afferenti. IMMUNOFLUORESCENZA: negativa.

Tali reperti erano compatibili con un quadro di grave nefroangiosclerosi (Fig. 4).

Il paziente è rimasto ricoverato per circa 10 giorni durante i quali è stata iniziata anche terapia nutrizionale con dieta ipoproteica $(0.6 \mathrm{~g} / \mathrm{kg}$ p.c.i. / die), ipofosforica, iposodica, e gli esami alla dimissione evidenziavano: creatininemia $2.7 \mathrm{mg} / \mathrm{dL}$, urea pl. $90 \mathrm{mg} / \mathrm{dL}$, proteinuria 2 $\mathrm{g} / 24 \mathrm{~h}$, clearance della creatinina $46 \mathrm{~mL} / \mathrm{min}$, emoglobina $10.3 \mathrm{~g} / \mathrm{LL}$, ematocrito $35 \%$. I valori di pressione si erano ridotti fino a $150 / 95 \mathrm{mmHg}$.

A distanza di circa 1 anno i valori di pressione arteriosa

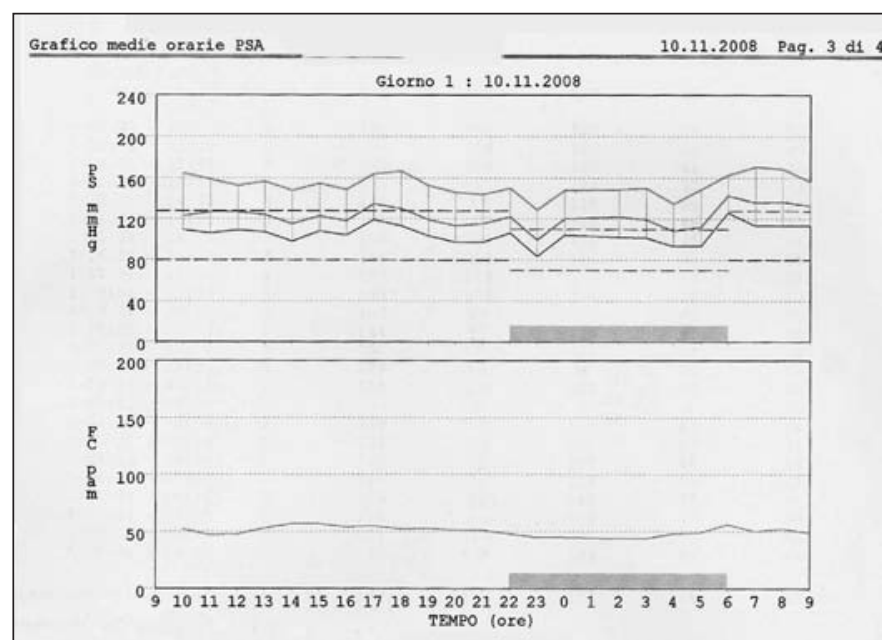

Fig. 2 - Registrazione pressoria nelle 24 ore precedenti la biopsia renale. Valori sistodiastolici elevati senza calo notturno.

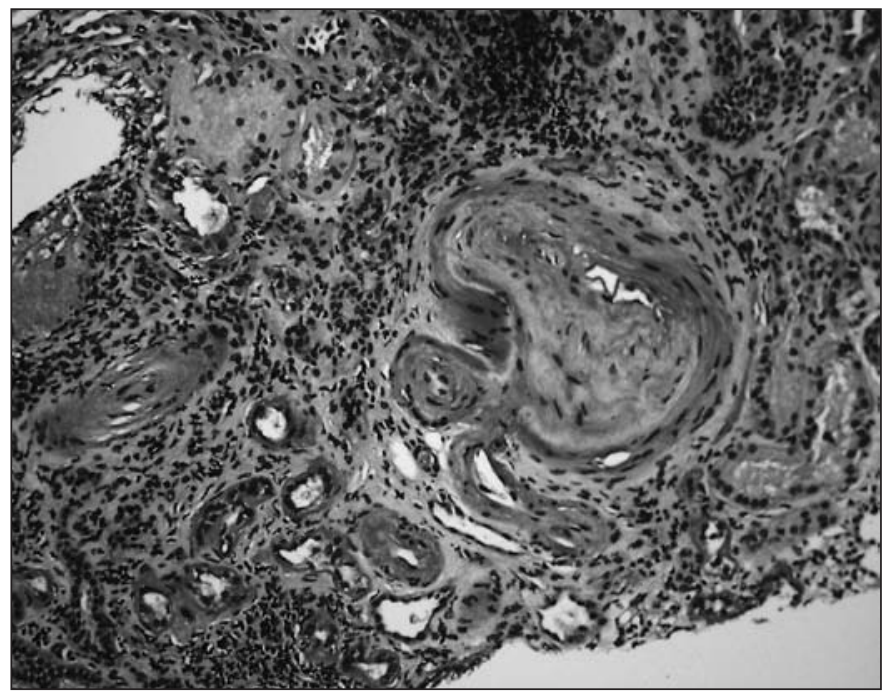

Fig. 4 - Esame istologico (colorazione PAS): quadro di grave nefrosclerosi.

sono meglio controllati, la funzione renale è migliorata con riduzione della proteinuria: creatininemia $2.3 \mathrm{mg} / \mathrm{dL}$, clearance della creatinina $54 \mathrm{~mL} / \mathrm{min}$, urea $\mathrm{pl} .70 \mathrm{mg} / \mathrm{dL}$, proteinuria $0.5 \mathrm{~g} / 24 \mathrm{~h}$, emoglobina $13.2 \mathrm{~g} / \mathrm{L}$, ematocrito $41 \%$, valori pressori medi di circa $135 / 85 \mathrm{mmHg}$; il paziente è rimasto asintomatico e non ha necessitato né di emotrasfusioni né di terapia con eritropoietina.

\section{Discussione}

Diverse patologie possono determinare un rapido deterioramento della funzione renale, e la sintomatologia talora può essere così sfumata che il paziente giunge 
all'osservazione con un'insufficienza renale non spiegabile. Le dimensioni e la struttura dei reni, evidenziabili con un esame ecografico, forniscono informazioni determinanti per un corretto inquadramento diagnostico (1). Oltre alla ricerca di segni di malattie sistemiche come il LES, le vasculiti e il mieloma, che talora si rivelano con questo quadro, il dato istologico è spesso determinante per una corretta gestione del paziente.

Controindicazioni assolute unanimemente riconosciute all'esecuzione della biopsia renale sono la presenza di anomalie della coagulazione, infezioni delle alte vie urinarie, ostruzione della via escretrice, malformazioni, e ipertensione severa (2).

Lipertensione maligna o accelerata si caratterizza per valori pressori elevati (pressione arteriosa diastolica superiore a $120 \mathrm{mmHg}$ ) accompagnati da emorragie retiniche, essudati o papilledema (3). Può svilupparsi interessamento renale, nefroangiosclerosi (4), ed in genere le forme aggressive tendono ad essere più comuni e più severe nei soggetti di razza nera (5).

Nel caso qui descritto, il paziente si è presentato con insufficienza renale di discreta entità, non in trattamento farmacologico, ed il quadro clinico (reni aumentati di volume e iperecogeni, proteinuria significativa, ipertensione arteriosa) ci ha indotto al sospetto di una nefropatia glomerulare a rapida evoluzione. Nei primi tre giorni di ricovero abbiamo cercato di ridurre i valori pressori con una terapia aggressiva con riduzione dei valori fino a $160 / 100 \mathrm{mmHg}$ al momento dell'agobiopsia renale (6). Il sanguinamento è la complicanza più frequente dell'agobiopsia renale (7). Valutazioni monocentriche su grandi numeri evidenziano una prevalenza di emorragia perirenale significativa di circa l' $8 \%(8,9)$. Il sanguinamento postbioptico può avvenire a tre livelli: a) all'interno delle vie urinarie, determinando ematuria macro o microscopica; b) sotto la capsula renale, determinando dolore e tamponamento renale; oppure c) nello spazio perinefrico, determinando ematoma e possibile anemizzazione nel giro di $12-24$ ore (10). Il paziente in effetti ha presentato un ematoma, raccoltosi nello spazio perirenale, dopo circa 5 ore dalla biopsia con progressivo aumento delle dimensioni che si sono stabilizzate dopo circa 2 giorni $(9 \mathrm{x} 4 \mathrm{x} 7 \mathrm{~cm})$ e che ha determinato un'importante anemizzazione.

Il paziente, senza la biopsia e/o la complicanza emorragica, probabilmente sarebbe stato sottoposto a terapia immunosoppressiva come nelle nefropatie rapidamente evolutive (11) e quindi sarebbe stato esposto inutilmente ai suoi potenziali effetti collaterali (12). Infatti, il quadro poteva avere le caratteristiche di una glomerulonefrite a rapida evoluzione.

In conclusione il caso presentato evidenzia come anche nel giovane di razza bianca l'ipertensione accelerata debba essere considerata tra le possibili cause di insufficienza renale proteinurica soprattutto quando i valori pressori elevati sono associati a segni di danno d'organo (fondo dell'occhio e cuore).

In tal caso appare prudente prima ottenere un adeguato controllo dei valori di pressione arteriosa e successivamente sottoporre il paziente alla biopsia renale che appare indispensabile per porre la diagnosi di certezza e per adottare il trattamento più idoneo alla patologia in atto $(6,13)$.

\section{Indirizzo degli Autori: \\ A. Capitanini, MD \\ U.O. Nefrologia ASL3 \\ Ospedale Civile \\ Via Cesare Battisti \\ 51017 Pescia (Pistoia) \\ drcapitanini@excite.it}

\section{Bibliografia}

1. Farrington K, Levison DA, Greenwood RN, Cattel WR, Baker RI. Renal biopsy in patients with unexplained renal impairment and normal kidney size. Quart J Med 1989; 263: 221-33.

2. Health and Public Policy Committee. American College of Physicians. Clinical competence in percutaneous renal biopsy. Ann Intern Med 1988; 108: 301-3.

3. Rosei EA, Salvetti M, Farsang C. European Society of Hypertension Scientific Newsletter: treatment of hypertensive urgencies and emergencies. J Hypertens 2006; 24: $2482-5$

4. Ahmed ME, Walker JM, Beevers DG, Beevers M. Lack of difference between malignant and accelerated hypertension. Br Med J 1986; 292: 235-7.

5. Cooper R, Rotimi C. Hypertension in blacks. Am J Hypertens 1997; 10: 804-12.

6. Appel GB. Renal biopsy: How effective, what technique, and how safe. J Nephrol 1993; 6: 4.

7. Eiro M, Katoh T, Watanabe T. Risk factors for bleeding complications in percutaneous renal biopsy. Clin Exp Nephrol 2005; 9: 40-5.

8. Stratta P, Canavese C, Marengo M, et al. Risk management of renal biopsy: 1387 cases over 30 years in a single centre. Eur J Clin Invest 2007; 37: 954-63. 
9. Meola M, Barsotti G, Cupisti A, Buoncristiani E, Giovannetti S. Free-hand uktrasound-guided renal biopsy: report of 650 consecutive cases. Nephron 1994: 67: 425-30.

10. Whittier WL, Korbet SM. Timing of complications in percutaneous renal biopsy. J Am Soc Nephrol 2004; 15: 142.

11. Couser WG. Rapidly progressive glomerulonephritis: Classification, pathogenetic mechanisms, and therapy. Am J Kidney Dis 1988; 11: 449-64.

12. Schacke H, Docke WD, Asadullah K. Mechanisms involved in the side effects of glucocorticoids. Pharmacol Ther 2002; 96: 23-43.

13. Madaio MP. Renal biopsy. Kidney Int 1990: 38: 529-43.

\section{TEST DI VERIFICA - 1}

1) Quali sono le complicanze più frequenti della biopsia renale oltre al sanguinamento?

a) Fistole artero venose parenchimali renali

b) Ipertensione arteriosa

c) Ematuria

2) Com'è l'indice di resistenza intraparenchimale nelle nefropatie glomerulari acute?

a) Aumentato

b) Normale

c) Ridotto

Le risposte corrette alle domande sono a pag. 51 